\title{
Photoelastic Phenomena in Stressed Laser Heterostructures
}

\author{
L.A. Kulakova*, N.S. Averkiev and A.V. Lutetskiy \\ Ioffe Physical Technical Institute, 26 Polytekhnicheskaya, St Petersburg 194021, Russian Federation
}

\begin{abstract}
The theoretical analysis of optical properties change in cubic crystals caused by simultaneous action of longitudinal and shear strains due to the photo-elastic interaction has been performed. The polarization characteristics change of a primary linear polarized light wave during propagation in the crystal has been studied. Results of the calculations were used to analyze photo-elastic phenomena in stressed heterostructures at the introduction of an alternating shear strain. The comparison of photo-elastic and acousto-electron phenomena in the laser heterostructures has been fulfilled. The results have been confirmed by the experiments in the InGaAs/GaAs structures at the action of shear strains excited by the ultrasonic waves.
\end{abstract}

DOI: $10.12693 /$ APhysPolA.127.87

PACS: 78.20.-e, 78.20.Bh, 78.20.hb

\section{Introduction}

It is known that influence of an ultrasonic strain on different objects is effective not only in applications, but also makes it possible to reveal and study new fundamental phenomena of solid state physics. Recently we have found (for the first time) that the ultrasonic strain can produce change in spectrum parameters of the laser heterostructures on a quantum well. They are (a) the frequency modulation of the optical emission, due to inphase action of the photo-elastic (PE) and the acoustoelectron (AE) interactions in the structures [1-3]; (b) the modulation of a laser emission direction due to the $\mathrm{PE}$ interaction [4]. In recent years, our study of polarization properties of the structures has revealed fundamental AE effect: the ultrasonic shear strain-induced modulation of the hole state energies in the quantum well. It leads to the alternating rotation of the emission polarization vector with the periodicity of the ultrasonic strain: $\phi_{a e}=\phi_{a e}^{0} \sin \Omega t$ [5]. However, despite of comprehensive experimental and theoretical validity of the effect, there is unexplored the question of possible influence of $\mathrm{PE}$ interaction on the polarization properties of the structures in the presence of an alternating shear strain. Effects of the induced piezo-birefringence become complicated because there are considerable intrinsic elastic stresses in quantum-dimensional heterostructures. This paper is devoted to the calculation of the polarization parameters change in a quantum-well heterolaser under simultaneous action of technological (longitudinal) and introduced shear strains. At the beginning the calculation in cubic crystals is fulfilled, because just the same symmetry is basic in the heterostructures. Then we use the obtained results to analyze PE effects arising in the structures under an ultrasonic shear strain action. At last we compare results of the calculations with the experimental data.

*corresponding author; e-mail: L.Kulakova@mail.ioffe.ru

\section{Rotation of principal axes under elastic strains}

Let us assume that a monochromatic light wave with linear polarization along $Y$ axis propagates in a cubic crystal along $X$ axis. At the presence of elastic strains in the polarization $Y Z$-plane tensor of dielectric permittivity becomes non-diagonal

$$
\begin{aligned}
& \varepsilon_{i k}=\left[\begin{array}{c}
\varepsilon_{22} \varepsilon_{23} \\
\varepsilon_{32} \varepsilon_{33}
\end{array}\right], \quad \varepsilon_{i k}=\varepsilon_{i k}^{0}+\Delta \varepsilon_{i k}, \\
& \Delta \varepsilon_{i k}=\varepsilon_{i} p_{i k m n} \varepsilon_{k} S_{m n},
\end{aligned}
$$

where $\varepsilon_{i k}^{0}=\delta_{i k} \varepsilon_{0}$ - dielectric constants in unstrained crystal, $p_{i k m n}$ - photo-elastic tensor components, $S_{m n}$ - strain tensor components. Due to the diagonalization we obtain (in matrix form) $\varepsilon_{i k}$ tensor eigenvalues $\lambda^{j}(j=1,2)$ in principal axes

$$
\begin{aligned}
\lambda^{j} & =\frac{2 \varepsilon_{0}+\varepsilon_{0}^{2}\left(p_{11}+p_{12}\right)\left(S_{2}+S_{3}\right)}{2} \\
& \pm \frac{\varepsilon_{0}^{2} \sqrt{\left(p_{11}-p_{12}\right)^{2}\left(S_{2}-S_{3}\right)^{2}+4 p_{44}^{2} S_{4}^{2}}}{2}
\end{aligned}
$$

and values of the direction cosines $A_{k}^{j}(k=2,3)$ of the principal axes.

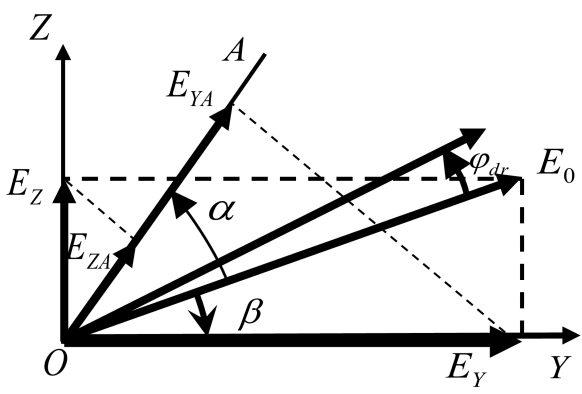

Fig. 1. Scheme of the change in the polarization vector direction with respect to the principal optical axes in the strained crystal.

To analyze the rotation of the principal axes, it is enough to consider the rotation angle $\beta$ (see Fig. 1) of $Y$ axis 


$$
\begin{aligned}
& A_{2}^{(2)}=\cos \beta, \\
& \cos \beta=\frac{1}{\sqrt{2}}\left(1+\frac{1}{\sqrt{1+B}}\right)^{1 / 2}, \\
& B=\frac{4 p_{44}^{2} S_{4}^{2}}{\left(p_{11}-p_{12}\right)^{2}\left(S_{2}-S_{3}\right)^{2}} .
\end{aligned}
$$

From (3) it is evident that at $S_{4}=0$ rotation of the principal axes is absent, $\beta=0$, but an optical anisotropy exists due to longitudinal strains in the crystal. Depending on the relation between strains $S_{i k}, 0>\beta \leq 45^{\circ}$. Results of the calculation, in accordance with $(3)$ ( $p_{i k}$ for $\mathrm{GaAs}$ crystals from [6]), are presented in Fig. 2. Main con-

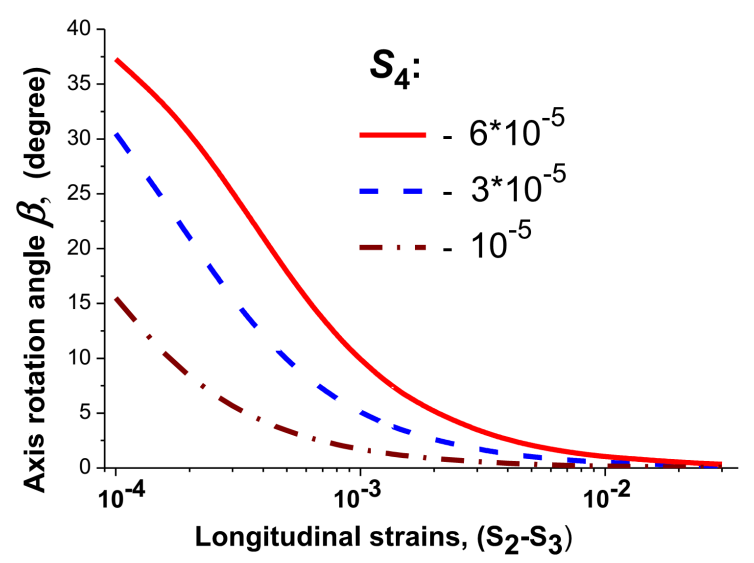

Fig. 2. Dependences of the $\beta$ on the longitudinal strain $\left(S_{2}-S_{3}\right)$ at different $S_{4}$.

clusions from the figure are as follows: (a) a significant rotation of the axes under strains occurs at comparable shear and longitudinal strains; (b) under conditions of strained quantum-size heterostructures, where $\left(S_{2}-S_{3}\right)$ is about $3 \times 10^{-2}$ and introduced strain $S_{4} \leq 10^{-4}$ [5], the parameter $B<4 \times 10^{-4}$, hence $\beta \ll 1$ :

$$
\beta \approx \frac{\sqrt{B}}{2}=\left|\frac{p_{44} S_{4}}{\left(p_{11}-p_{12}\right)\left(S_{2}-S_{3}\right)}\right| .
$$

\section{Rotation of the polarization vector}

At the rotation of the principal axes on the angle $\beta$, polarization vector of a linear polarized wave (with amplitude $E_{0}$ ) can be presented as a sum of two waves with mutually perpendicular $E_{Y}$ and $E_{Z}$ polarization directions (Fig. 1). Refractive indexes $n_{Y}$ and $n_{Z}$ for them in the stressed crystal are different. So the phase difference $\delta$ :

$$
\begin{aligned}
& \delta=2 \pi L\left(n_{Y}-n_{Z}\right) / \lambda_{0}, \\
& \left(n_{Y}-n_{Z}\right)=\left(\lambda^{Y}-\lambda^{Z}\right) / 2 n_{0}
\end{aligned}
$$

appears at the crystal output due to double refraction (dr). Using Eq. (2), we obtain

$$
\delta=\left(\pi L n_{0}^{3} / \lambda_{0}\right) \sqrt{\left(p_{11}-p_{12}\right)^{2}\left(S_{2}-S_{3}\right)^{2}+4 p_{44}^{2} S_{4}^{2}},
$$

where $\lambda_{0}$ is the wavelength of light in vacuum and $L$ is the crystal length. The ellipticity of the output polarization is determined by the $\delta$, and the output intensity
$\left(I \propto E^{2}\right)$ can be presented as follows:

$$
\begin{aligned}
I= & I_{0}\left[\sin ^{2} \beta^{*} \sin ^{2}(\alpha+\beta)+\cos ^{2} \beta^{*} \cos ^{2}(\alpha+\beta)\right. \\
& \left.+\frac{1}{2} \sin 2 \beta^{*} \sin 2(\alpha+\beta)^{*} \cos \delta\right] .
\end{aligned}
$$

The light wave intensity reaches extreme values along the ellipse axes. So from $I_{\alpha}^{\prime}=0$ we obtain the rotation angle $\alpha_{\text {extrem }}=\phi_{\mathrm{dr}}$ of the direction of the ellipse axes with respect to the initial direction of the maximum intensity in the crystal (Fig. 1):

$$
\tan 2 \phi_{\mathrm{dr}}=\frac{(\cos \delta-1) \tan 2 \beta}{1+\cos \delta^{*} \tan ^{2} 2 \beta} .
$$

Expression (8) allows the following conclusions.

(i) Dependence of $\phi_{\mathrm{dr}}$ on the strain values has periodic character (Fig. 3), which is determined by the value of $\delta$.

(ii) Value of the $\phi_{\mathrm{dr}}$ is independent of the sign of strain combination $\left(S_{2}-S_{3}\right)$ and $S_{4}$, since both the $\delta$ and the $\beta$ are defined by magnitudes of these strains.

(iii) So an alternating strain (for example, $S_{4} \propto \sin \Omega t$ ) leads to change in time of $\phi_{\mathrm{dr}}$ with double periodicity in comparison with alternating strain.

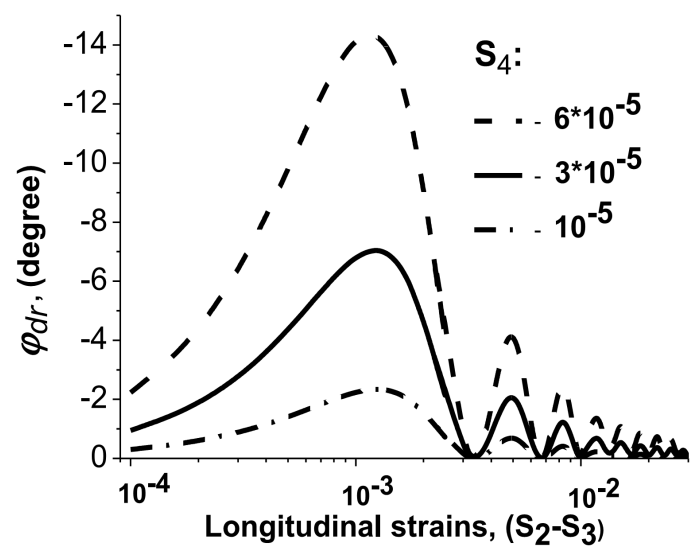

Fig. 3. Dependences of the $\phi_{\mathrm{dr}}$ on the longitudinal strain $\left(S_{2}-S_{3}\right)$ at different $S_{4}$.

The expression (8) is possible to simplify neglecting the second order small terms in $\beta$ (the case in the structures of our interest):

$$
\begin{aligned}
& I=I_{0}\left[\cos ^{2} \alpha+A \sin 2 \alpha\right], \\
& A=(\cos \delta-1) \beta \approx 1 / 2 \sin 2 \phi_{\mathrm{dr}} .
\end{aligned}
$$

The first term in (9) describes the angular dependence of the output intensity of linearly polarized radiation. The second term in (9) formally looks as a consequence of the polarization vector rotation in the strained crystal by the angle $\phi_{\mathrm{dr}}$.

\section{Experimental results and discussion}

Alternating strains were produced by introduction of bulk acoustic waves. We studied the case, when a sound wave $S=S_{0} \sin (Q z-\Omega t)$ propagates across a thin semiconductor layer that means almost constant spatial distribution of the elastic strain across an optical resonator: 
$S \approx S_{0} \sin \Omega t$. The polarization analysis has been carried out in two laser structures with two different compositions, operating at room temperatures in pulse mode. Details of the experimental setup are presented in $[2,5]$.

(i) In GaAsP $/ \operatorname{InP}$ - heterolasers $\left(\lambda_{0}=1.49 \mu \mathrm{m}\right)$. The shape of the equilibrium emission pulse is close to rectangular. Angular dependence of its intensity $I_{=}=$ $A=\cos ^{2} \alpha$ shows that the laser under study provides linear polarization. The introduction of sound leads to the modulation of the intensity with the wave periodicity: $I_{\sim}=I_{\sim}^{0}(\alpha) \sin \Omega t$. Angular dependence of the amplitude is well described by the expression

$$
I_{\sim}^{0}(\alpha)=A_{\sim}^{0} \cos ^{2} \alpha+B_{\sim}^{0} \sin 2 \alpha .
$$

In the experiments $A_{\sim}^{0}, B_{\sim}^{0} \ll A_{=}$, so the total intensity can be written as

$$
I(\alpha)=I_{=}+I_{\sim}^{0} \approx A_{=}\left[\cos ^{2} \alpha+\left(B_{\sim}^{0} / A_{=}\right) \sin 2 \alpha\right],
$$

From the experimental data we have obtained the value of $\phi_{a e}^{0} \approx\left(B_{\sim}^{0} / A_{=}\right)=1.2^{\circ}$. The theoretical analysis shows that the rotation of the polarization direction is due to mixing of light and heavy hole wave functions in the laser quantum well. The calculation of the $\phi_{\mathrm{dr}}\left(p_{i k}\right.$ for GaAs-crystals [6]) has shown that $\phi_{\mathrm{dr}}^{0}$ does not exceed $4 \times 10^{-4} \mathrm{rad}(0.025 \mathrm{deg})$. So $\phi_{\mathrm{dr}}^{0} \ll \phi_{a e}^{0}$. It is possible reason that we could not reveal $\mathrm{PE}$ contribution in the light modulation intensity in these structures.

(ii) InGaAs/GaAs heterolasers $\left(\lambda_{0}=1.06 \mu \mathrm{m}\right)$. The laser equilibrium emission consists of two components $E^{Y}$ and $E^{Z}$ polarized along $Y$ and $Z$ axis.

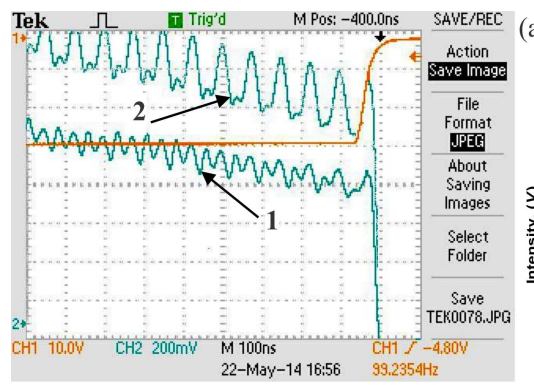

a) (b)

Fig. 4. (a) Superimposition of two oscillograms: top beam (orange line) - laser operating current; bottom beams - the intensities of the emission in the presence of the ultrasonic strain, with the frequency of $20 \mathrm{MHz}:$ (1) $\alpha=82^{\circ}$, (2) $\alpha=79^{\circ}$; (b) results of the calculation according to (12): (1) $\alpha=82^{\circ},(2) \alpha=79^{\circ}$.

The introduction of the sound wave also leads to the modulation of both intensity components. Because $E^{Z} \ll E^{Y}$ it has become possible to reveal alternating component with double periodicity of the sound. It is demonstrated by oscillograms in Fig. 4a. Time dependence of total intensity $I(\alpha, t)$ including the $(\mathrm{dc}) I_{=}(\alpha)$ and the $(\mathrm{ac})$ $I_{\sim}(\alpha, t)$ signals are well approximated by the expressions

$$
\begin{aligned}
& I(\alpha, t)=I_{=}(\alpha)+I_{\sim}(\alpha, t) ; \\
& I_{=}(\alpha)=\left(E^{Y} \cos \alpha-E^{Z} \sin \alpha\right)^{2},
\end{aligned}
$$

$$
\begin{aligned}
& I_{\sim}(\alpha, t)=\left[I_{Y \sim}^{0} \cos ^{2}(\alpha)-I_{Z \sim}^{0} \sin ^{2}(\alpha)+I_{a e \sim}^{0} \sin (2 \alpha)\right] \\
& \times \sin (\Omega t)+I_{d r \sim \sin }^{0}(2 \alpha)|\sin (\Omega t)| .
\end{aligned}
$$

First term in (12) arises from the modulation intensity with periodicity of the sound wave; the second one has double periodicity of the sound wave and is due to induced piezobirefringence. Result of the calculation of $I(\alpha, t)$ at $\alpha=82^{\circ}$ and $\alpha=79^{\circ}$ with $I_{Y=}=$ $I(0)=44.5 \mathrm{~V}, I_{Z=}=I(90)=0.55 \mathrm{~V}, I_{Y \sim}^{0}=1.8 \mathrm{~V}$, $\left.I_{Z \sim}^{0}=0.306 \mathrm{~V}, I_{\text {ae } ~}^{0}=1.016 \mathrm{~V}, I_{\mathrm{dr} \sim}^{0}=0.44 \mathrm{~V}\right)$ is presented in Fig. 4b. On the basis of the results obtained we have got the values of $\phi_{a e}^{0} \approx 1.3 \mathrm{deg}$ and $\phi_{\mathrm{dr}}^{0}=0.56 \mathrm{deg}$. Increase of $\phi_{\mathrm{dr}}^{0}$, in our opinion, is due to both larger internal strain values and higher efficiency of the PE interaction in the InGaAs/GaAs heterostructures.

\section{Conclusions}

(i) The rotation of the polarization vector of radiation, propagating through the strained laser heterostructure, is possible within both the $\mathrm{AE}$ and the $\mathrm{PE}$ mechanisms if shear strain is introduced into it.

(ii) Manifestations of these mechanisms essentially differ: rotation angles vary in time with different periodicities: (a) $\phi_{a e}(t) \propto \sin \Omega t$; (b) $\phi_{\mathrm{dr}}(t) \propto|\sin \Omega t|$. This factor apparently makes it possible to separate contributions of these two mechanisms to the polarization change.

(iii) The study performed has shown reliably that the polarization vector direction modulation $\left(\phi_{a e}(t) \propto\right.$ $\sin \Omega t$ ) under ultrasonic shear strain, detected in InGaAsP/InP-heterolasers, indeed results from the fundamental quantum effect of mixing of wave functions of hole states in the quantum well due to the AE interaction.

(iv) The rotation of the emission polarization with double periodicity of sound $\left(\phi_{\mathrm{dr}}(t) \propto|\sin \Omega t|\right)$, revealed in the InGaAs/GaAs heterolasers, reliably confirmed the possibility of efficient influence of the PE interaction on the polarization properties of a laser emission in the presence of shear strains.

\section{Acknowledgments}

This work was supported by the RFBR (Grant No. 11-02-00729) and scientific programs of the Russian Academy of Sciences (Programme No. 24 and 27).

\section{References}

[1] L.A. Kulakova, I.S. Tarasov, JETP Lett. 78, 67 (2003).

[2] L.A. Kulakova, N.A. Pikhtin, S.I. Slipchenko, I.S. Tarasov, JETP 104, 689 (2007).

[3] L.A. Kulakova, Appl. Opt. 48, 1128 (2009).

[4] L.A. Kulakova, A.V. Lyutetskii, V.B. Voloshinov, Tech. Phys. Lett. 36, 563 (2010).

[5] L.A. Kulakova, N.S. Averkiev, A.V. Lyutetskiy, V.A. Gorelov, Semiconductors 47, 135 (2013).

[6] R.W.J. Dixon, J. Appl. Phys. 38, 5149 (1967). 